\title{
Estudio de la difusión pulmonar de monóxido de carbono en enfermos con estenosis mitral
}

\author{
C. ZAMARRÓN SANZ, F. DEL CAMPO MATÍAS, A. AMARO CENDÓN*, \\ M. SALGUEIRO RODRÍGUEZ, J.R. RODRÍGUEZ SUÁREZ
}

Servicio de Neumología. *Servicio de Cardiología. Hospital Clínico Universitario. Santiago de Compostela. Servicio de Neumología. Hospital Río Hortega.Valladolid

\author{
PULMONARY DIFFUSION CAPACITY STUDY IN MITRAL VALVE \\ STENOSIS PATIENTS
}

\begin{abstract}
RESUMEN
Objetivo: Estudiar la difusión pulmonar de monóxido de carbono en enfermos con estenosis mitral.

Método: Se estudiaron 15 sujetos control y 15 pacientes con estenosis mitral. A todos ellos se les realizó una espirometría forzada y un test de difusión pulmonar con CO (DLCO) por el método de la respiración única con determinación del volumen sanguíneo capilar pulmonar $(\mathrm{Vc})$ y del factor de difusión de membrana $(\mathrm{Dm})$. Además, a los pacientes con estenosis mitral se les practicó un estudio ecocardiográfico-doppler.

Resultados: Los pacientes con estenosis mitral presentaron un aumento de la DLCO y del Vc y una disminución de la relación $\mathrm{Dm} / \mathrm{Vc}$, estando el $\mathrm{Vc}$ relacionado inversamente con el área valvular mitral $(\mathrm{r}=-0,63 ; \mathrm{p}=0,037)$. No se observó correlación entre el resto de los parámetros cuantificados por ecocardiografia-doppler y los obtenidos en el estudio de función pulmonar. Tampoco se observaron diferencias entre ambos grupos en el factor de difusión de membrana.

Conclusiones: Los pacientes con estenosis mitral presentan un incremento de la difusión pulmonar de monóxido de carbono y del volumen sanguíneo capilar pulmonar sin cambios en el factor de difusión de membrana.
\end{abstract}

PALABRAS CLAVE: Estenosis mitral. Capacidad de difusión pulmonar. Pruebas de función pulmonar.

\begin{abstract}
Objective: The aim of our work has been the study of $\mathrm{CO}$ diffusion capacity in mitral valve stenosis patients.

Method: We have studied 15 control subjects and 15 patients with mitral valve stenosis. We performed spirometry study and CO pulmonary diffusion tests (DLCO) by single breath method to determine two components: pulmonary capillary blood volume $(V c)$ and membrane diffusion factor $(\mathrm{Dm})$. In addition, in mitral valve stenosis patients we performed a ecocardiography-doppler study.

Results: The mitral valve stenosis group had higher values of DLCO and $V c$ and a lower ratio of $D m / V c$ than the control group. There is a negative correlation between $V c$ and the mitral valve area $(r=-0.63$; $p=0.037)$. We do not find any another correlation between the rest of ecocardiography doppler parameters and lung function test variables that we have measured. We have not found any differences between both groups in Dm.

Conclusions: Mitral valve stenosis patients present a increase of $C O$ pulmonary diffusion capacity and pulmonary capillary blood volume without changes in membrane diffusion factor.
\end{abstract}

KEY WORDS: Mitral valve stenosis. Pulmonary diffusing capacity. Lung function tests.

Zamarrón Sanz C, del Campo Matías F, Amaro Cendón A, Salgueiro Rodríguez M, Rodríguez Suárez JR. Estudio de la difusión pulmonar de monóxido de carbono en enfermos con estenosis mitral. An Med Interna (Madrid) 2002; 19: 66-68.

\section{INTRODUCCIÓN}

La estenosis mitral es una enfermedad valvular cardíaca que dificulta en mayor o menor grado el flujo diastólico desde al aurícula izquierda al ventrículo izquierdo. Esta enfermedad provoca una elevación de la presión venosa pulmonar, de forma pasiva, con aumento subsiguiente de la presión arterial pulmonar pudiendo generar cambios en la estructura y función de este órgano (1). Se ha descrito una disminución de la compliance pulmonar (2), desequilibrios en la relación ventilación y percusión (3), obstrucción de vías aéreas periféricas (4) y alteraciones de la capacidad de difusión de monóxido de carbono (DLCO) $(5,6)$. Todos estos trastornos se han puesto en relación con las alteraciones hemodinámicas producidas por esta enfermedad valvular, mejorando al instaurar el tratamiento de la enfermedad (7-10).

Utilizando métodos invasivos, Bjure y col. determinaron el volumen sanguíneo capilar pulmonar y la DLCO en diferentes enfermedades cardiopulmonares (11). No obstante dado que, según las fórmulas aportadas por Roughton y Forster (12), la DLCO está condicionada por la resistencia

Trabajo aceptado: 26 de octubre de 2001

Correspondencia: C. Zamarrón Sanz. Servicio de Neumología. Hospital Clínico Universitario. C/ Travesía de la Choupana s/n. 15706. Santiago de Compostela. 
de la membrana alveolo-capilar (Dm) y del volumen capilar pulmonar ( $\mathrm{Vc}$ ) así como por la afinidad del monóxido de carbono con la hemoglobina, es posible calcular la Dm y Vc de forma no invasiva (13). Sin embargo, existen escasos estudios sobre las variaciones de ambos factores en enfermos con estenosis mitral (5) y no conocemos ninguno que relacione estas variables con parámetros ecocardiograficos y hemodinámicos medidos por doppler, en enfermos hemodinámicamente estables.

El objetivo de nuestro trabajo ha sido el estudio de la difusión pulmonar de $\mathrm{CO}$ en pacientes con estenosis mitral.

\section{PACIENTES Y MÉTODOS}

Hemos estudiado 15 pacientes con estenosis mitral, sin historia previa de cirugía valvular cardíaca o de enfermedad pulmonar primaria. El grupo control lo constituyen 15 sujetos, dentro del mismo rango de edades al grupo de estudio, sin alteraciones relevantes en la radiografía de tórax y ausencia de antecedentes de enfermedad cardiopulmonar. Todos ellos eran no fumadores.

Se practicó un estudio de función pulmonar mediante un equipo computarizado que consistía en una curva de flujo volumen y un test de difusión pulmonar de monóxido de carbono siguiendo la metodología descrita previamente (14).

A los pacientes del grupo con estenosis mitral se les realizó el estudio eco-doppler midiendo la máxima velocidad del flujo auriculo-ventricular y calculando el gradiente trasmitral máximo y medio así como el área valvular mitral. Además se determinó la fracción de eyección del ventrículo izquierdo (15).

Todos los pacientes estaban estables, en ritmo sinusal y presentaban fracciones de eyección del ventrículo izquierdo superiores al $50 \%$.

\section{ANÁLISIS ESTADÍSTICO}

Los datos se expresan en medias \pm DE. Para la comparación de los valores (antropométricos, espirométricos y de difusión pulmonar de $\mathrm{CO}$ ) entre los grupos a estudio se ha utilizado la prueba $\mathrm{t}$ de Student o la prueba de la $\mathrm{U}$ de Mann-Whitney. Para estudiar la relación entre variables continuas se ha utilizado el coeficiente de correlación de Spearman. Se consideran significativos aquellos con valores de $\mathrm{p}<0,05$.

\section{RESULTADOS}

Los datos antropométricos así como los resultados de la espirometrías se exponen en la tabla I. No se observaron diferencias significativas entre pacientes con estenosis mitral y controles en las variables estudiadas.

Los parámetros obtenidos del estudio de difusión pulmonar de $\mathrm{CO}$ en ambos grupos se exponen en la tabla II. Los pacientes con estenosis mitral presentan un incremento de la DLCO, Vc, Vc/Va, así como una disminución de la relación $\mathrm{Dm} / \mathrm{Vc}$, con respecto al grupo control. Aunque los valores del Dm son superiores a los del grupo control estos no alcanzan significación estadística.
TABLA I

CARACTERÍSTICAS ANTRO PO M ÉTRICAS Y VALO RES DE LAS VARIABLES ESPIROM ÉTRICAS DE LOS DIFERENTES GRUPOS

\begin{tabular}{lccc}
\hline & $\begin{array}{c}\text { Controles } \\
(n=15)\end{array}$ & $\begin{array}{c}\text { Estenosis mitral } \\
(n=15)\end{array}$ & $p$ \\
\hline Edad (años) & $51,1 \pm 15,8$ & $51,3 \pm 10,8$ & ns \\
Altura (cm) & $163,5 \pm 2,9$ & $160,6 \pm 11,3$ & ns \\
Sexo maculino (n) & 7 & 7 & ns \\
FVC (L) & $3,0 \pm 0,7$ & $2,8 \pm 1,0$ & ns \\
FVC (t) (\%) & $94,6 \pm 7,8$ & $86,0 \pm 16,2$ & ns \\
FEV $_{1}(\mathrm{~L} / \mathrm{s})$ & $2,5 \pm 0,6$ & $2,6 \pm 0,9$ & ns \\
FEV $_{1}$ (t) (\%) & $102,4 \pm 12,8$ & $100,8 \pm 16,3$ & ns \\
FEV $_{1} / \mathrm{FVC} \mathrm{( \% )}$ & $82,7 \pm 6,8$ & $90,1 \pm 5,6$ & ns \\
FEF $_{25-75 \%}$ & $2,9 \pm 0,9$ & $3,3 \pm 1,5$ & ns \\
FEF $_{25-75 \%}(\mathrm{t})(\%)$ & $97,3 \pm 21,0$ & $104,7 \pm 31,3$ & ns \\
\hline
\end{tabular}

FVC: Capacidad vital forzada. FVC (t) (\%): Porcentaje del valor teórico; $\mathrm{FEV}_{1}$ : Volumen espiratorio forzado en el primer segundo. $\mathrm{FEV}_{1}(\mathrm{t})(\%)$ : Porcentaje del valor teórico. $\mathrm{FEF}_{25-75 \%}$ : Flujo espiratorio forzado entre el 25 y el $75 \%$ de la capacidad vital forzada. $\mathrm{FEF}_{25.75 \%}$ (t) (\%): Porcentaje del valor teórico. ns: No significativo.

\section{TABLA II}

VARIABLES O BTENIDAS EN EL ESTUDIO DE DIFUSIÓN PULM ONAR EN LOS DIFERENTES GRUPOS

\begin{tabular}{lccc}
\hline & $\begin{array}{c}\text { Controles } \\
(n=15)\end{array}$ & $\begin{array}{c}\text { Estenosis mitral } \\
(n=15)\end{array}$ & $p$ \\
\hline VA (L) & $4,1 \pm 1,0$ & $4,5 \pm 1,2$ & ns \\
DLCO $(\mathrm{ml} / \mathrm{min} / \mathrm{Hg})$ & $24,1 \pm 4,3$ & $28,0 \pm 6,0$ & 0,004 \\
DLCO (t) $(\%)$ & $95,5 \pm 11,5$ & $101,7 \pm 25,7$ & $\mathrm{~ns}$ \\
$\mathrm{DLCO} / \mathrm{VA}$ & $5,9 \pm 0,8$ & $6,4 \pm 0,6$ & 0,000 \\
$\mathrm{Dm}(\mathrm{ml} / \mathrm{min} / \mathrm{Hg})$ & $27,8 \pm 7,1$ & $29,0 \pm 8,4$ & $\mathrm{~ns}$ \\
$\mathrm{Dm} / \mathrm{VA}$ & $6,7 \pm 1,4$ & $6,5 \pm 0,9$ & $\mathrm{~ns}$ \\
$\mathrm{Vc}(\mathrm{ml})$ & $104,1 \pm 121,3$ & $265,4 \pm 111,3$ & 0,003 \\
$\mathrm{Vc} / \mathrm{VA}$ & $32,3 \pm 49,8$ & $56,5 \pm 24,6$ & 0,002 \\
Dm/Vc & $0,46 \pm 0,2$ & $0,13 \pm 0,02$ & $\mathrm{~ns}$ \\
\hline
\end{tabular}

VA: Volumen alveolar. DLCO : Difusión pulmonar de CO. DLCO (t) (\%): Porcentaje del valor teórico Vc: Volumen sanguíneo capilar pulmonar. Dm: Difusión pulmonar de membrana. ns: No significativo.

Los pacientes con estenosis mitral presentaban una velocidad máxima de $1,4 \pm 0,5 \mathrm{~m} / \mathrm{s}$, gradiente trasmitral máximo de $8,7 \pm 5,8 \mathrm{mmHg}$, gradiente trasmitral medio de $3,15 \pm$ $1,4 \mathrm{mmHg}$, área valvular mitral de $1,7 \pm 0,35 \mathrm{~cm}^{2}$ y una fracción de eyección de $0,73 \pm 0,1$.

Se observó una correlación negativa significativa entre el área valvular mitral y el volumen sanguíneo capilar pulmonar $(r=-0,63, p=0,037)$. No se detectó ninguna otra correlación entre el resto de los parámetros cuantificados por ecocardiografía-doppler y los obtenidos en el estudio de función pulmonar. 


\section{DISCUSIÓN}

En este estudio se observa que los pacientes con estenosis mitral presentan un incremento del la DLCO y del volumen sanguíneo capilar pulmonar el cual se correlaciona inversamente con el área valvular mitral.

La estenosis mitral es una enfermedad crónica que origina cambios vasculares y del tejido intersticial pulmonar (1). Estos pacientes presentan un aumento lento de la presión capilar pulmonar con producción de edema intersticial y peribronquial que puede alterar la difusión pulmonar de monóxido de carbono $(5,6)$. Además, se ha descrito un incremento la resistencia de la vía aérea atribuido a hiperreactividad bronquial (16-18) y que remite al realizar valvuloplastia percutánea (19).

Las alteraciones en la difusión pulmonar de $\mathrm{CO}$ en pacientes con estenosis mitral se relacionan con la situación hemodinámica del enfermo en el momento del estudio así como a la fase evolutiva de la enfermedad valvular. En un grupo de pacientes con fallo cardiaco, Faggiano y col. (20) encontraron una reducción de la DLCO y de la Dm, comparado con los sujetos normales, con un volumen capilar dentro del rango de la normalidad. Esta disminución de la DLCO, debido a la caída del factor de difusión de membrana, se atribuyó a edema intersticial.
En nuestro estudio, no se observan diferencias en el FEF $25-75 \%$ ni en el factor de difusión de membrana, con respecto al grupo control. Esta ausencia de alteraciones en las vías aéreas pequeñas y en la membrana podría relacionarse con la lenta instauración de los cambios hemodinámicos en el flujo pulmonar en la estenosis mitral así como a la ausencia de fallo cardiaco asociado.

Por otra parte, nuestros pacientes presentan un incremento del Vc con respecto al grupo control, que se relaciona inversamente con el área valvular mitral. Dado que el Vc depende de las relaciones entre las presiones de la arteria pulmonar, aurícula izquierda y alvéolos (5); la disminución del área valvular mitral aumentaría la presión dentro de la aurícula izquierda explicando el incremento del Vc y de la DLCO.

Algunos estudios han sugerido que la DLCO se incrementa en los estadios iniciales de la enfermedad, debido al aumento del Vc, con caída en los estadios avanzados asociados a una disminución de la Dm (16,20-22). Por tanto podemos asumir que nuestros enfermos se encontraban en una fase inicial de la evolución de la estenosis mitral, con cambios unicamente en el Vc.

Concluimos que el estudio de la DLCO con cálculo del volumen sanguíneo capilar pulmonar y del factor de difusión de membrana puede aportar información relevante para el estudio de los pacientes con estenosis valvular mitral.

\section{Bibliografía}

1. Cortese DA. Pulmonary function in mitral stenosis. Mayo Clin Proc 1978; 53: 321-326.

2. Wood TE, McLeod P, Anthonisen NR, Macklem PT. Mechanics of breathing in mitral stenosis. Am Rev Respir Dis 1971; 104: 52-60.

3. Dawson A, Rocamora JM, Morgan JKR. Regional lung function in chronic pulmonary congestion with and without mitral stenosis. Am Rev Respir Dis 1976; 113: 51-59.

4. Yoskioka T, Nakanishi N, Okubo S, Kunieda T, Ishikura F, Nagata S. Improvement in pulmonary function in mitral stenosis after percutaneous transvenous mitral commissurotomy. Chest 1990; 98: 290-294.

5. Burgess JH. Pulmonary diffusing capacity in disorders of the pulmonary circulation. Circulation 1974; 49: 541-550.

6. Werner FM, Kolmer HHB. The CO single breath transfer factor of the lung. A reliable clinical parameter in valvular heart disease. Respiration 1981; 41: 155-165.

7. Levine MJ, Weinstein JS, Diver DJ, Berman AD, Wyman RM, Cunningham MJ, Safian RD, Grossman W, Mackay RG. Progressive improvement in pulmonary vascular resistance after percutaneous mitral valvuloplasty. Circulation 1989; 79: 1061-1067.

8. Yoshioka T, Nakanishi N, Okubo S, Kunieda T, Ishikura F, Nagata S. Improvement in pulmonary function in mitral stenosis after percutaneous trasvenous mitral commissurotomy. Chest 1990; 90: 290-294.

9. Ray S, Dodds P, Wilson G, Morrison L, Walshaw M, Perry R. Effects of balloon mitral commissurotomy on the diffusing capacity of the alveolar capillary membrane and pulmonary capillary volume in patients with mitral stenosis. Am J Cardiol 1994; 15: 1068-1070.

10. Gomez-Hospital JA, Cequier A, Romero PV, Canete C, Ugartemendia C, Mauri J, Esplugas E Partial improvement in pulmonary function after successful percutaneous balloon mitral valvotomy. Chest 2000; 117: 643-648.

11. Bjure J, Korsgren M, Varnauskas E. Pulmonary blood volume and diffusing capacity in cardio-pulmonary disease. Clin Sci 1967; 33: 225-232.

12. Roughton FJW, Forster RE. Relative importance of diffusion and chemical reaction rates in determining rate of exchange of gases in the human lung with special reference to true diffusing capacity of pulmo-

nary membrane and volume of blood in the lung capillaries. J Appl Physiol 1957; 11: 290-302.

13. Cotes JE. Measurement of transfer factor (Diffusing capacity) and its subdivisions. In: Lung function. Assessment and application in medicine. 5th ed. Oxford: Blackwell Scientific Publications 1993.

14. Zamarrón C, Del Campo F, Paredes C, Rodríguez Suárez JR. Estudio de la difusión pulmonar de monóxido de carbono en dos situaciones clínicas: asma bronquial y diabetes mellitus. An Med Interna (Madrid) 2001: 18: $237-242$.

15. Feigenbaum H. Echocardiography in Heart Disease, 4th ed, Philadelphia, Saunders, 1992; 81.

16. Cabanes LR, Weber SN, Matran R, Regnard J, Richard MO, Degeorges ME, Lockhart A. Bronchial hyperresponsiveness to methacoline in patients with impaired left ventricular function. N Engl J Med 1989; 320: $1317-1322$.

17. Nishimura Y, Maeda H, Yokoyama M, Fukuzaki H. Bronchial hyperreactivity in patients with mitral valve disease. Chest 1990; 98: 1085-1090.

18. Rolla G, Bucca C, Brussino L, Bugiani M, Bergerone S, Malara D, Morea M. Bronchial responsiveness, oscillations of peak flow and symptoms in patients with mitral stenosis. Eur Respir J 1992; 5: 213-218.

19. Gülec S, Ertas F, Tutar E, Demirel Y, Karaoguz R, Omurlu K, Oral D. Bronchial hiperreactivity in patients with mitral stenosis before and after successful percutaneous mitral ballon valvulotomy. Chest 1999; 116: $1582-1586$

20. Faggiano P, D'Aloia A, Simoni P, Gualeni A, Foglio K, Ambrosino N Giordano A. Effects of body position on the carbon monoxide diffusing capacity in patients with chronic heart failure: relation to hemodynamic changes. Cardiology 1998; 89: 1-7.

21. Rhodes KM, Evemy K, Nariman S, Gibson GJ. Relation between severity of mitral valve disease and results of routine lung function tests in non-smokers. Thorax 1982; 37: 751-755.

22. Jebavy P, Hurych J, Widimsky J. Influence of pulmonary hypertension on pulmonary diffusing capacity in patients with mitral stenosis. Respiration $1978 ; 35: 1-7$. 